\title{
Considerations for Physical Facility Design and Management of a State-of-the-Art Electron Microscopy and Analysis Laboratory
}

\author{
Daniel E. Huber ${ }^{1}$, Jonathan Orsborn ${ }^{1}$, Henk O. Colijn ${ }^{1}$, Cameron Begg ${ }^{1}$, Michael J. Mills ${ }^{1}$, David W. \\ McComb $^{1}$, Hamish L. Fraser ${ }^{1}$ \\ 1. Center for Electron Microscopy and Analysis, Materials Science and Engineering, The Ohio State \\ University, Columbus, OH, U.S.A.
}

Modern electron microscopes demand a physical environment to enable them to achieve or even surpass the manufacturers performance specifications. This can prove to be formidable challenge to facility managers and researchers. Factors that commonly influence the performance of electron microscopes include, but are not limited to temperature fluctuations, air currents and pressure changes, electromagnetic fields, vibrational and acoustic disturbances. However, with proper considerations given to design and implementation of the physical microscopy facility, the influence of all of these factors can be mitigated. The Center for Electron Microscopy and Analysis (CEMAS), at The Ohio State University, opened in September 2013, was designed with the goal of optimizing the environment for state of the art microscopy.

The following paper will address common issues relevant to facility mangers. Then describe how these issues are addressed at CEMAS. Firstly, we will address environmental concerns, temperature stability, electromagnetic fields, vibrational stability and grounding. However, these issues can be tied closely to the second effort related to building automation and physical plant design. Finally, a facility needs to address the possible loss of utility power and minimize the possibility of down instrument time.

With these critical issues addressed the obvious problem becomes managing the volume of data produced in a large multi user facility. A brief discussion on the implementation of virtualized environment for computing and data storage capabilities at CEMAS. Where the design of a facility wide network can address security, connectivity and the management of computing resources and related workflows. CEMAS has also adopted Facility Online Manager to handle, scheduling and billing activities. The close coupling of the scheduling and billing activities increases facility efficiency by allowing instrument managers to focus on instrumentation, research and training.

The result of these efforts in designing an optimal environment can be seen in the following Figure 1. This figure shows the installation of an FEI Titan 80-300 in a purpose built instrument room, where operators of the microscope is performed from a joining control room. The operator controls are simply transmitted from the instrument room to the control room. This configuration isolates the instrument from the operator and promotes high environmental stability. These levels of environmental stability are a complement to one with low EMI. To achieve a site with very low EMI carful characterization of the site of was necessary. Field survey work prior to construction concluded that nearby power lines, shown being demolished in Figure 2, induced current in building steel and would negatively affect instrument performance. The relocation effort significantly reduced levels of EMI observed within the facility and has greatly contributed to the success of the facility. 


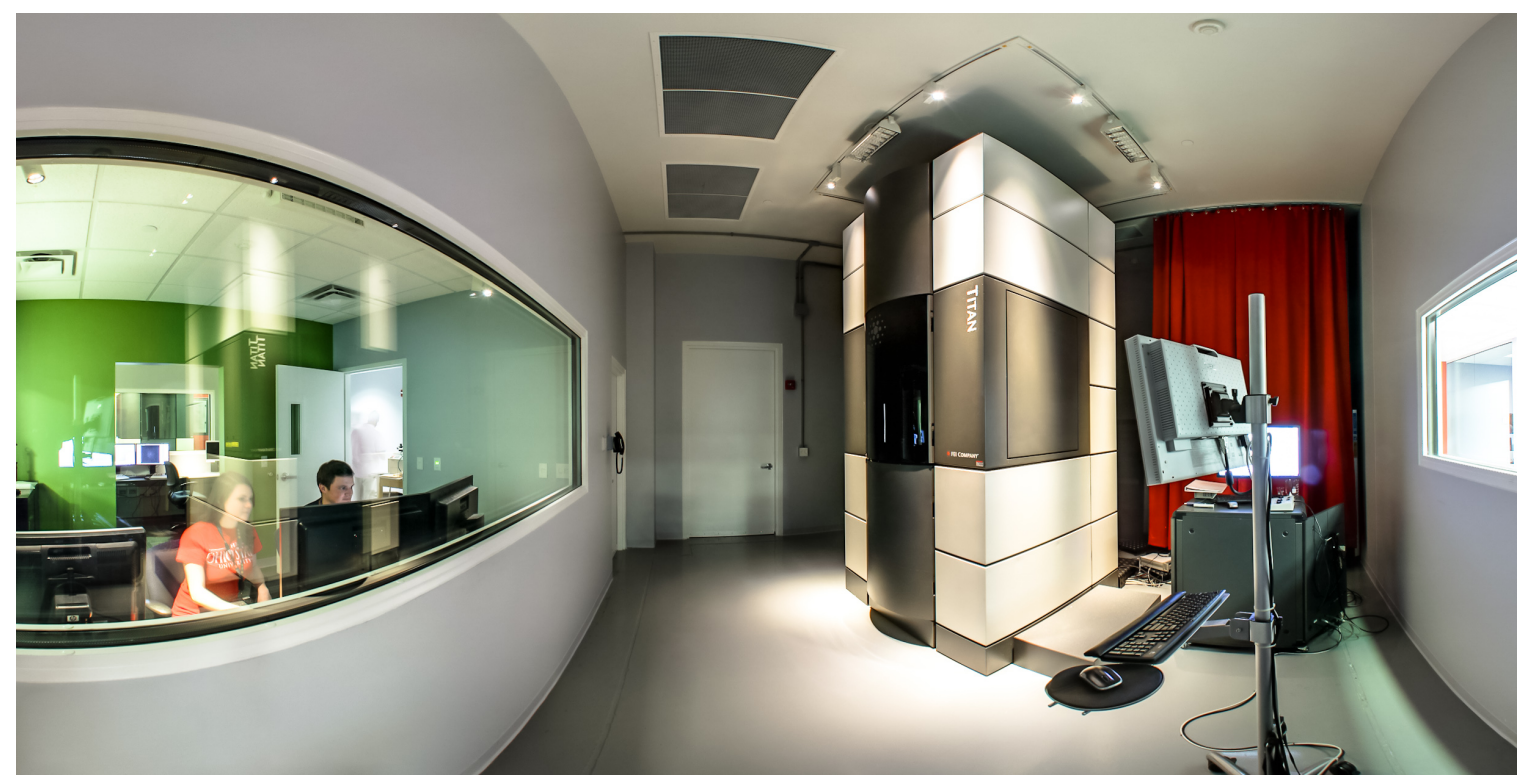

Figure 1. FEI Titan 80-300 installed at CEMAS at The Ohio State University.

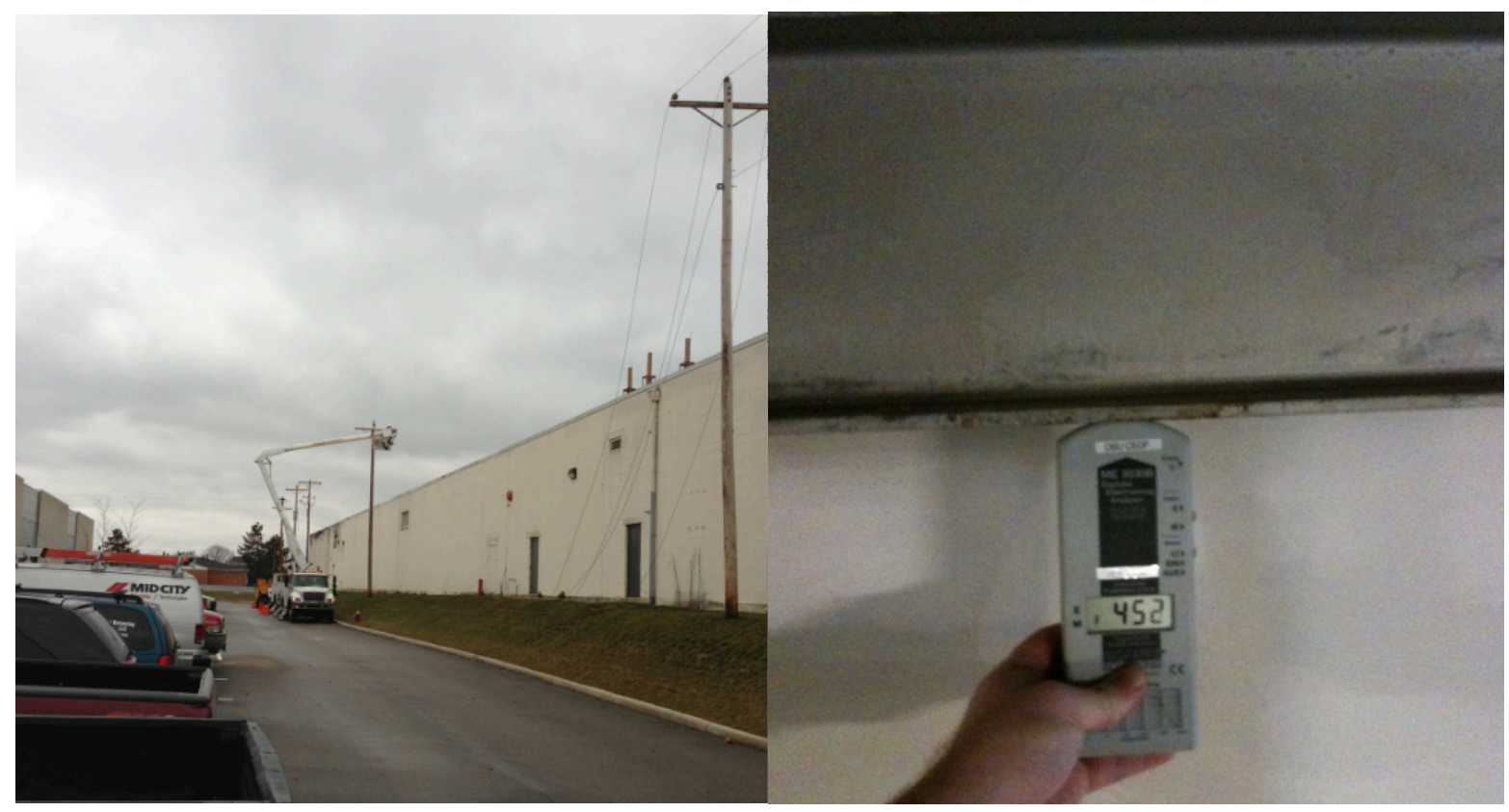

Figure 2. Relocation of power lines adjacent to new CEMAS, performed by local utility (Left). Magnetic fields from power lines inducing current in building steel as evidenced by field survey work (Right). 